\title{
Gibt es effiziente Herangehensweisen zur Vermittlung und Aneignung von Sprachgeschichtskenntnissen für den Bedarf der Grundschullehrer?
}

\author{
Mojmír Muzikant
}

The article deals with advantages and disadvantages of several university textbooks in the area of history of German language. Then, the new textbook "Short history of German Language for Students of Teaching" is introduced and the typology of exercises is outlined.

history of German language - textbooks analysis - didactic application - exercise typology

Im vorliegenden Aufsatz werden Vor- und Nachteile einiger Handbücher für das Fach ,Deutsche Sprachgeschichte' kommentiert. Anschließend wird ein neu erarbeitetes Lehrbuch ,Kurze Geschichte der deutschen Sprache für Lehramtsstudenten Deutsch als Fremdsprache vorgestellt und eine Typologie der enthaltenen Übungsreihe umrissen.

deutsche Sprachgeschichte - Analyse von Lehrmaterialien - didaktische Umsetzung Übungstypologie

\section{Das Fach ,Sprachgeschichte` in Studienplänen der Auslandsgermanistik}

Die diachrone Betrachtungsweise der Sprache macht es möglich, dass die Studierenden den gegenwärtigen Sprachzustand nicht als etwas Unveränderliches, Erstarrtes ansehen, sondern ihn im Gegenteil als eine zeitweilige Größe betrachten, die aus dem Zusammenwirken von x-historischen Aspekten hervorgegangen und stets in Entwicklung begriffen ist. Und gerade das Gewinnen dieser Einsicht ist sehr wichtig, weil viele Studierende mehr oder weniger im Allgemeinen dazu neigen, die Gegenwartssprache als die einzige, für immer gültige Norm ohne Zulassung anderer Möglichkeiten wahrzunehmen.

\section{Bewertung der bestehenden Lehrmaterialen}

Für Kurse im Bereich, Sprachgeschichte` gibt es ein fast unübersehbares Angebot an Lehrmaterialen. Nicht alle sind jedoch für den Bedarf unseres künftigen Deutschlehrers schon aus Gründen des verfügbaren Stundenpensums optimal. Ich möchte an dieser Stelle keine ausführliche Analyse von Lehrmaterialen vornehmen, vielmehr sollen meine Vorbehalte an drei Beispielen konkretisiert werden.

Das erste Lehrbuch, das ich hier kurz ansprechen will, ist die 2007 bereits in 10. Auflage erschienene, von Helmut Langner überarbeitete, Geschichte der deutschen Sprache' (SCHMIDT: 2007). Der präsentierte Lehrstoff ist für unsere Zwecke zu umfangreich und unübersichtlich, nicht zuletzt auch deswegen, weil die 
Ausführungen in zwei selbständige Abschnitte eingeteilt sind. Im ersten werden die historisch-gesellschaftlichen und sprachpolitischen Hintergründe behandelt, im zweiten dann die herkömmlichen sprachlichen Charakteristiken einzelner Entwicklungsetappen auf der lautlichen, morphologischen und syntaktischen Sprachebene. Die Darlegungen sind von Textbeispielen begleitet, allerdings fehlt es an Material zur Einübung und Festigung der erworbenen Kenntnisse.

Beim zweiten Beispiel handelt es sich um das ,Arbeitsbuch Alt- und Mittelhochdeutch' des Autorenkollektivs PAULY/BERGMANN/FANKHÄNEL (1999), das neben übersichtlichen und zutreffenden morphologischen und lautlichen Darlegungen auch Textproben und Übungen einbezieht. Von Nachteil ist jedoch die Tatsache, dass eine effektive Arbeit mit dem Übungsteil die Heranziehung eines althochdeutschen (ahd.) bzw. mittelhochdeutschen (mhd.) Wörterbuchs notwendig macht.

An dritter Stelle ist hier das Lehrmaterial ,Historische Entwicklung des Deutschen ‘ (MASAŘÍK/BROM: 2005) zu nennen. Es handelt sich um Übersichtstabellen mit knappen Kommentaren, die als Einführung in das Studium genügen könnten, zumal im Anhang auf zusätzliche Internet-Materialen hingewiesen wird. Die drei kurzen Textproben (ahd., mhd., frnhd.) sind mit Interpretationshilfen versehen. Eine selbständige Arbeit mit dem Lehrmaterial anhand grammatischer und lexikalischer Kommentare ist jedoch m. E. auch nicht möglich. Übungen zur Festigung der historischen Laut- und Formenlehre bleiben aus.

\section{3. Übungstypologie für neu zu erstellende Lehrtexte}

Aus dem oben Gesagten ergibt sich logischerweise die Konsequenz, zur Beseitigung der kritisierten Schwachstellen beizutragen. Einen Lösungsweg sehe ich in der Erarbeitung neuer Lehrtexte, die dem angedeuteten Defizit Rechnung tragen. Im Folgenden soll in Kürze die Übungstypologie vorgestellt werden, die ich für mein eigenes Skriptum (MUZIKANT: im Druck) gewählt habe. Zu jeder Übung wird dabei im Anhang auch eine Lösung beigegeben, damit die Lernenden mit dem Material auch im Selbststudium arbeiten können. Es muss hinzugefügt werden, dass nicht alle Kapitel und Unterkapitel die gleichen Möglichkeiten zur didaktischen Umsetzung bieten.

Sehr geeignet zur Einübung ist das aus dem Indoeuropäischen ererbte Ablautsystem, auf dem die unregelmäßigen Verben basieren. Insgesamt wurden zu diesem Thema sechs Übungen mit folgendem Aufbau erarbeitet.

In Übung VI wird die Bildung von Stammformen der Verben der ersten Klasse (Ablautreihe) sowie die Bestimmung der nhd. Lautgestalt des Infinitivs eingeübt. Dieselbe Aufgabe ist in der Übung VII zu lösen, in der die Verben der 2. Klasse behandelt werden. In Übung VIII werden die Stammformen der dritten Klasse (Präs., Sg./Pl. Prät., Part.) mit ihren nhd. Entsprechungen verglichen. Der Lerner 
soll hier erkennen, dass einige von den angeführten Verben ihre starken Formen zum Nhd. verändert haben bzw. zu den schwachen Verben übergegangen sind. In Übung IX haben die Lerner die Aufgabe, starke Verben (Klassen 4 bis 6) in kleinen Textproben zu suchen, ihre Formen (grammatischen Kategorien) zu bestimmen und sie den entsprechenden Klassen zuzuordnen. In Übung X sind die ursprünglich reduplizierenden Verben der siebenten Klasse zusammengefasst. Die Lerner sollen entscheiden, bei welchen der angeführten Verben sich vollständig oder zum Teil schwache Formen durchgesetzt haben. In Übung XI wird auf die Liste der starken Verben in der DUDEN-Grammatik (2005) verwiesen. Die Lerner haben die Aufgabe zu bestimmen, bei welchen starken Verben der Stammvokal des Konj. Prät. auf die dritte Stammform des Ahd./Mhd. zurückgeht. Der Bildung des schwachen Präteritums ist dagegen nur eine Übung (Übung XII) gewidmet.

Aus dem ahd. Lautstand wird die Aufmerksamkeit auf die 2. Lautverschiebung gerichtet (Übung V). In einem aktuellen Text in Plattdeutsch sollen die Lerner Wörter und Wortverbindungen mit unverschobenen Verschlusslauten suchen und die hochdeutschen Entsprechungen anführen. Die mhd. Neuerungen im Lautstand sowie z. T. auch in der Morphologie gegenüber dem Ahd. sollen die Lerner anhand der angeführten ahd. Wortformen rekonstruieren (Übung XIII). Ferner sollen auch mhd. Paradigmen zu den angeführten ahd. Deklinations- und Konjugationsmustern gebildet werden (Übung XIV). Auf dieselbe Art und Weise werden auf der Basis des Mhd. die frnhd. Neuerungen erklärt und geübt (Übung XV).

Bei einigen der erarbeiteten Übungen wird die diachrone Dimension im Grunde genommen weniger berücksichtigt. Dies ist bei der Einübung von Wortpaaren (Zwillingsformeln) und doppelförmigen Verben der Fall. Diese sprachlichen Erscheinungen sind der Gegenwartssprache eigen. Geschichtlich gehen sie aber auf das urgermanische Merkmal ,Festlegung des Wortakzents auf den Wortanfang zurück, das u. a. auch in der Alliteration seinen Niederschlag findet. Weil diese Phänomene den Studierenden nicht selten Schwierigkeiten bereiten, habe ich mich entschieden, auch diese in die Übungsreihe aufzunehmen (Übungen I, II, III, IV).

Darüber hinaus wird in den Lehrtexten häufig mit Textbeispielen gearbeitet. Die wichtigsten sprachlichen Unterschiede werden anhand einer Gegenüberstellung des Gotischen und seiner ahd. mhd. und frnhd. Entsprechungen demonstriert. Außer den ahd., mhd. und frnhd. Textproben mit Übersetzungen ins Nhd. werden auch Texte aus dem ahd. Tatian zur selbständigen Analyse mit grammatischer und lexikalischer Stütze angeboten.

\section{Proben aus den Übungen zu den starken Verben}

\section{Übung VI}

Bilden Sie zu den angegebenen ahd. Infinitiven die übrigen Stammformen. Versuchen Sie, die Lautgestalt des Infinitivs im Nhd. zu bestimmen: 
1. slìdan, 2. scrîtan, 3. strîtan, 4. bîzan usw.

\section{Übung VII}

Ergänzen Sie zu den angegebenen ahd. Infinitiven die übrigen Stammformen. Versuchen Sie, die Lautgestalt des Infinitivs im Nhd. zu bestimmen:

1. liogan, 2. fiogan, 3. kriochan, 4. biotan usw.

\section{Übung VIII}

Welche von den angeführten ahd. starken Verben der dritten Klasse weisen in ihren nhd. Stammformen (Präsensstamm, Präteritum, Partizip) Abweichungen auf:

1. rinnan, 2. swimman, 3. dringan, 4. findan usw.

\section{Übung IX}

Suchen Sie in den folgenden Textproben starke Verben, bestimmen Sie ihre Formen (gramm. Kategorien) und versuchen Sie, diese der entsprechenden Klasse zuzuordnen:

O 1,4,21: Thar gisah er stantan gotes boton sconan. T 85, 4: uelfa azzant fon brosmun usw.

\section{Übung X}

2. waltan ,walten', 3. fâhan ,fangen', 4. hâhan ,hängen', 5. slâfan ,schlafen' usw. Versuchen Sie, die übrigen Stammformen zu bilden. Bei welchen von den genannten Verben haben sich im Nhd. zum Teil bzw. vollständig schwache Formen durchgesetzt?

\section{Übung XI}

Bei welchen von den starken Verben kann der Stammvokal des Konjunktivs Präteritum auf die ursprüngliche dritte Stammform (Pl. Prät.) zurückgeführt werden? (Vgl. die Liste starker/unregelmäßiger Verben, DUDEN: Die Grammatik 2005, S. 491-502.)

\section{Perspektiven}

Abschließend sei bemerkt, dass die Effizienz der Veranstaltung davon abhängt, inwieweit es gelingt, sprachliche Gegebenheiten einzelner Entwicklungsetappen an Textbeispielen anzuwenden und Bewegungen im Wortschatz und der Grammatik bis zum Neuhochdeutschen anschaulich zu demonstrieren. $\mathrm{Ob}$ das einzusetzende Lehrbuch die gehegten Erwartungen erfüllt, muss allerdings die Unterrichtspraxis erst noch bestätigen. 


\section{Literatur:}

BERGMANN/PAULY/MOULIN-FANKHÄNEL (1999): Bergmann, Rolf/Pauly, Peter/ Moulin-Fankhänel, Claudine. Alt-und Mittelhochdeutsch: Arbeitsbuch zur Grammatik der älteren deutschen Sprachstufen und zur deutschen Sprachgeschichte. Göttingen: Vandenhoeck \& Ruprecht, 5. Aufl., 1999.

DUDEN (2005): Die Grammatik. Mannheim: Bibl. Inst., 7. Aufl., 2005.

MASAŘÍK/BROM (2005): Masař́k, Zdeněk/Brom, Vlastimil. Historische Entwicklung des Deutschen. Übersichtstabellen - Kommentare - Textproben. Brno: Masaryk-Universität, 2005.

MUZIKANT (im Druck): Muzikant, Mojmír. Kurze Geschichte der deutschen Sprache für Lehramtsstudenten,Deutsch als Fremdsprache؛ Brno: Masaryk-Universität.

SCHMIDT (2007): Schmidt, Wilhelm. Geschichte der deutschen Sprache. Ein Lehrbuch für das germanistische Studium. Stuttgart; Leipzig: Hirzel, 10. Aufl. unter Leitung von Helmut Langner und Norbert Richard Wolf, 2007. 